\title{
Nearly asymptomatic intracranial capillary hemangiomas: A case report and literature review
}

\author{
XIAOYU XIA ${ }^{1}$, HAOJU ZHANG ${ }^{1}$, HONGYAN GAO ${ }^{2}$, YI YANG ${ }^{1}$, \\ YIWU DAI $^{1}$, YANG JIAO ${ }^{3}$ and JIANGHONG HE ${ }^{1}$ \\ ${ }^{1}$ Department of Neurosurgery; ${ }^{2}$ Medical Administration Division; ${ }^{3}$ Department of Stomatology, \\ PLA Army General Hospital, Beijing 100700, P.R. China
}

Received September 15, 2016; Accepted May 11, 2017

DOI: $10.3892 / \mathrm{etm} .2017 .4780$

\begin{abstract}
The present study reported a nearly asymptomatic case of intracranial capillary hemangioma (ICHs), which are rare benign vascular tumors or tumor-like lesions. A 33-year-old female came to the hospital with a complaint of a slight but recurring morning headache concentrated in the left posterior occipital area. These headaches spontaneously resolved without any treatment. Computed tomography and magnetic resonance imaging revealed a mass inside the left occipital lobe. The patient refused to undergo conservative observation at home and insisted on radical therapy. Prior to surgery, an atypical meningioma or astrocytoma was suspected. A navigation-guided brain-mass resection was performed under general anesthesia and a solid mass closely associated with the tentorium cerebelli was completely resected. Histopathological analysis confirmed diagnosis of an $\mathrm{ICH}$. The patient recovered well and experienced no major neurological defects, apart from an issue with the right visual field. The present study also conducted a retrospective literature review of papers published in English describing cases of intracranial capillary hemangiomas. A PubMed search identified 19 articles comprising 29 cases. The clinical symptoms of $\mathrm{ICH}$ are diverse and all reported cases in the literature were symptomatic. Previous studies demonstrated that diagnoses of intracranial capillary hemangioma are usually made during surgical resection by histopathological examination. Treatment for ICH remains empirical and surgery is the most common method of treatment. Patient prognosis is generally good-the majority of patients achieve long-term, event- and progression-free survival.
\end{abstract}

Correspondence to: Professor Jianghong He, Department of Neurosurgery, PLA Army General Hospital, 5 Nanmencang, Dongsishitiao Street, Beijing 100700, P.R. China

E-mail: jiaxy02@163.com

Key words: intracranial capillary hemangioma, intracranial, diagnosis, asymptomatic, literature review

\section{Introduction}

Capillary hemangiomas $(\mathrm{CHs})$ are benign vascular tumors or tumor-like lesions that usually occur at birth or in early infancy. Typically, the lesions involve skin and soft tissue, and are usually found on the face, scalp, chest, or back $(1,2)$. They have been reported to occur in 1.1 to $2.6 \%$ of full-term neonates, with an estimated frequency of 10 to $12 \%$ within the first year of life (2). They are characterized by a proliferative phase followed by an involutionary phase and patients often experience spontaneous regression with age $(2,3)$. Lesions can occur in adults, occur more often in women than men and undergo changes in size relative to hormonal cycles and pregnancy (2).

CHs involving the nervous system are rare (4) and primarily occur in the spinal nerve roots or cauda equina $(5,6)$. Intracranial capillary hemangiomas (ICHs) are even rarer; only 29 cases have been reported so far, however the intracranial location of these lesions varies. All reported cases were confirmed histologically however no patients were diagnosed prior to pathological examination. Clinical experience of this disease is poor and the lesion is usually considered to be a meningioma prior to surgery (7). Due to the small number of reported cases, the true prevalence of ICHs may be underestimated as they may remain asymptomatic or undergo spontaneous regression (3).

The current study reported a case of ICH in a patient admitted to the Department of Neurosurgery, PLA Army General Hospital (Beijing, China). Furthermore, previous studies investigating the diagnosis, differential diagnosis with other brain tumors such as meningiomas, and treatment for ICHs were reviewed.

\section{Case report}

A 33-year-old woman attended the Department of Neurosurgery, PLA Army General Hospital in December 2014 with the complaint of a minor morning headache for 7 days that was concentrated in the left posterior occipital area. The headache was alleviated following rest. The patient had suffered from mild hypertension for nearly 5 years since her last pregnancy but her blood pressure was well controlled with oral medication of amlodipine benzenesulfonate (Pfizer, Inc., 
New York, NY, USA; $10 \mathrm{mg}$ a day). The patient was examined at the outpatient clinic of the Department of Neurosurgery, PLA Army General Hospital and underwent computed tomography (CT) and magnetic resonance imaging (MRI) scans, which revealed a mass inside the left occipital lobe (Figs. 1 and 2). The patient refused conservative observation at home and insisted on undergoing radical therapy, and was subsequently admitted to the Department of Neurosurgery, PLA Army General Hospital on March 9th, 2015. Although no medical treatment was given to the patient prior to surgery, the patient experienced no further headaches and no fever, nausea or vomiting. Following admittance to hospital, examinations identified no signs of neurological damage. All the aspects of the present study complied with the Declaration of Helsinki, 1995. Written informed consent was obtained from the patient and family members and the present study was authorized by the Ethics Committee of the Department of Neurosurgery, PLA Army General Hospital (Beijing, China).

Atypical meningioma or astrocytoma was suspected. A routine cerebral angiography was performed through the right femoral artery puncture with an Artis Zeego angiographic system (Siemens AG, Munich, Germany). Iodixanol injection was used as the contrast agent. This revealed a hypervascular lesion that was primarily supplied by the meningohypophyseal trunk; however, no evident draining vein was identified (Fig. 3). A navigation-guided brain-mass resection was performed under general anesthesia. Sufentanil (IDT Biologika GmbH, Dessau-Rosslau, Germany; $25 \mu \mathrm{g}$ ), propofol (AstraZeneca, Cambridge, UK; $100 \mathrm{mg}$ ) and rocuronium bromide (N.V. Organon; Merck KGaA, Darmstadt, Germany; $50 \mathrm{mg}$ ) were used to induce anesthesia. Propofol (AstraZeneca, $200 \mathrm{mg} / \mathrm{h}$ ) and remifentanil (Yichang Humanwell Pharmaceutical Co., Ltd., Yichang, China; $1,000 \mu \mathrm{g} / \mathrm{h}$ ) were used to maintain anesthesia. High intracranial pressure was detected during surgery. At first, entry was attempted through the tentorium cerebelli space to reduce brain damage but this failed. Eventually the occipital cortex was perforated to get to the tumor directly.

A solid mass closely associated with the tentorium cerebelli was completely resected. Intraoperative bleeding was kept under control and no substantial bleeding was encountered; however, an infratentorial epidural hematoma formed following surgery. This was identified $24 \mathrm{~h}$ after surgery in the routine CT exam, requiring a second operation of hematoma evacuation (Fig. 4). The patient recovered well with no major neurological defects apart from a half visual field defect (right temporal side). An MRI scan performed $\sim 2$ months later revealed no recurrence of the tumor (Fig. 5); however, the right visual field defect persisted and the patient is being observed in the outpatient clinic every 3 months.

Histopathological analysis. Histopathological analysis confirmed diagnosis of ICH. On visual inspection, the completely resected tumor was similar to a dark-red mulberry with multiple prominences. It was a firm, solid, 2.2- x 1.6- x 1.7-cm mass with a broad-based dural attachment (Fig. 6A). Sectioning identified a dark-grey, oozing cut surface.

The tumor tissue was fixed with $10 \%$ neutral buffered formalin at room temperature for $72 \mathrm{~h}$. Sections $(4 \mu \mathrm{m})$ were stained with hematoxylin and eosin and examined under a

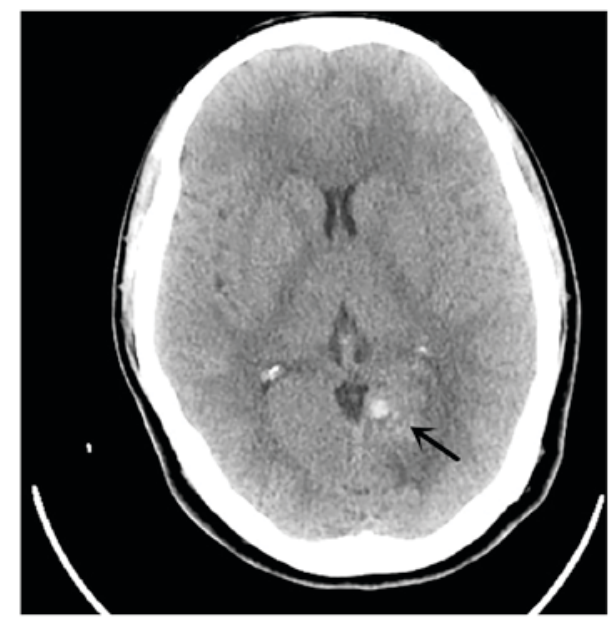

Figure 1. Computed tomography of the patient's brain prior to surgery. A high-density, round mass (indicated by the arrow was detected with a diameter of $\sim 2.0 \mathrm{~cm}$ and areas of higher density inside the left occipital lobe. Edema was observed along the edges.

light microscope (Olympus BX53; Olympus Corporation, Tokyo, Japan). Microscopic examination revealed a vascular malformation with thin-walled and delicate vessels, which varied greatly in size from a small lumina lined with plump endothelial cells to dilated vessels lined with flattened endothelium; red blood cells were observed within the vessel lumens (Fig. 6B). No intervening brain tissues or hemosiderin deposits were identified. There was no evidence of nuclear atypia, significant mitotic activity or cytoplasmic hyaline globules and no morphological evidence of metastasis.

Immunohistochemistry. Immunohistochemical staining was performed using the EnVision system (Agilent Technologies, Inc.). Briefly, serial 5- $\mu$ m-thick sections were cut from formalin-fixed and paraffin-embedded tumor blocks, dewaxed in xylene, rehydrated through sequential changes of alcohol, and then antigen retrieved using $0.01 \mathrm{M}$ citrate buffer, $\mathrm{pH} 6.0$, at $90^{\circ} \mathrm{C}$ for $20 \mathrm{~min}$. After the tissue sections were washed with PBS, they were incubated with fresh $3 \%$ hydrogen peroxide for $20 \mathrm{~min}$ at room temperature. The sections were then blocked with $20 \%$ goat serum for $30 \mathrm{~min}$ and incubated with the following primary antibodies for $2 \mathrm{~h}$ at room temperature: Anti-EMA (ab156947; 1:150 dilution; Abcam, Cambridge, UK), anti-progesterone receptor (ab32085; 1:100 dilution; Abcam), anti-S100 (ab14849; 1:3,000 dilution; Abcam), anti-SOX2 (ab92494; 1:100 dilution; Abcam), anti-GFAP (ab7260; 1:1,000 dilution; Abcam), anti-NeuN (ab177487; 1:3000 dilution; Abcam) anti-Olig2 (ab109186; 1:100 dilution; Abcam), anti-CD31 (ab28364; 1:50 dilution; Abcam), anti-CD34 (ab81289; 1:2500 dilution; Abcam) and anti-vimentin (ab28028; 1:100 dilution; Abcam). The sections were then incubated with a secondary antibody Envision FLEX+ (K8002; Agilent Technologies, Inc.) for $20 \mathrm{~min}$ at room temperature. Immunostaining was finally developed with 3,3-diaminobenzidine. Positive and negative controls were run as appropriate. The sections were assessed independently by two experienced pathologists. Immunostaining with epithelial membrane antigen, progesterone receptor, S-100, sex determining region Y-box 2, glial fibrillary acidic protein, 


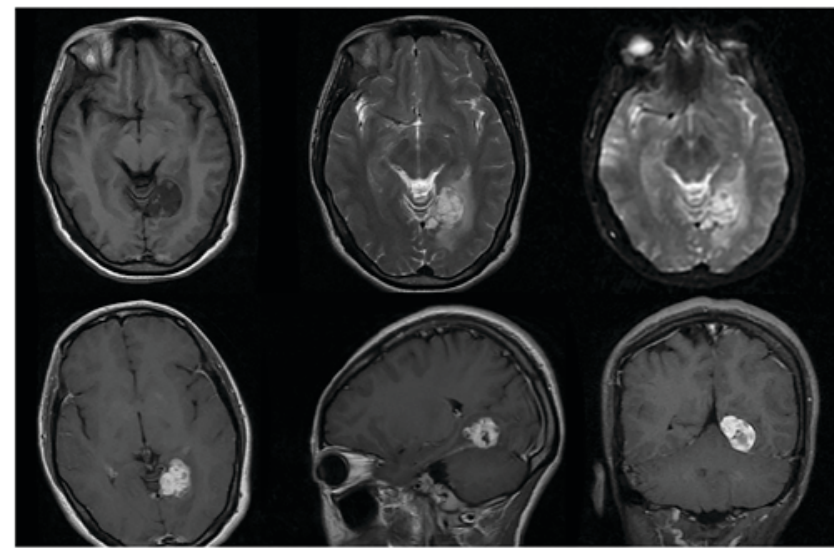

Figure 2. Brain magnetic resonance imaging of the current patient prior to surgery. A mass with heterogeneous, long T1 and T2 signals located inside the left occipital lobe and was near the posterior horn of the left ventricle close to the tentorium. Marked uneven reinforcement was observed when enhanced.

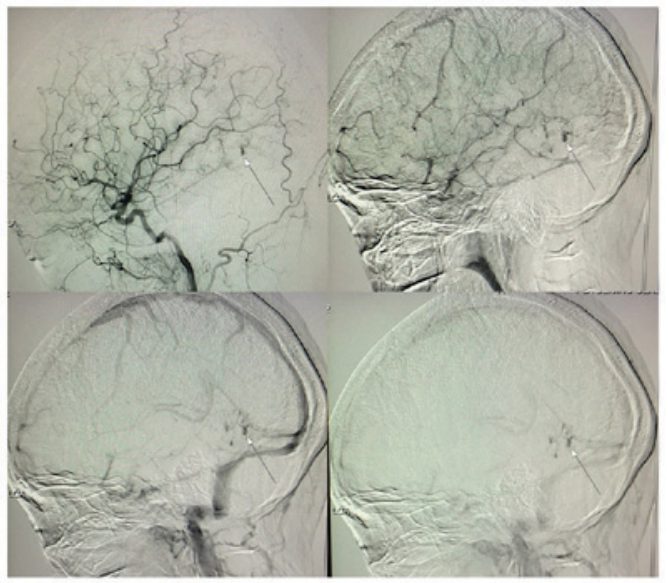

Figure 3. Cerebral vascular angiography of the current patient prior to surgery. Abnormal architecture of tumor vascularity was detected, including chaotic distribution, tumor staining and pooling of contrast material. Clear time of contrast agent at tumor area was $\sim 5 \mathrm{sec}$ later than other areas. The arrow indicates tumor location.

neuronal nuclei and Oligo-2 (all Abcam) exhibited negative results, while immunostaining with cluster of differentiation 31 (CD 31), CD 34 and vimentin (all Abcam exhibited strong positive results, which implied vascular proliferation (Fig. 6C and D). The vascular nature of the lesion was confirmed and a diagnosis of meningioma was ruled out.

\section{Discussion}

$\mathrm{CHs}$ are relatively common benign vascular tumors that occur in $1.0-2.6 \%$ of live births and are generally located in the skin and soft tissues (8). These tumors are more common in females than males and may undergo changes in size according to hormonal fluctuations and pregnancy (2). CHs involving the central nervous system are rare. The majority of documented cases have been identified in the spinal roots and the cauda equina. ICHs are even more rare. In the current study, a search was conducted using the query 'intracranial

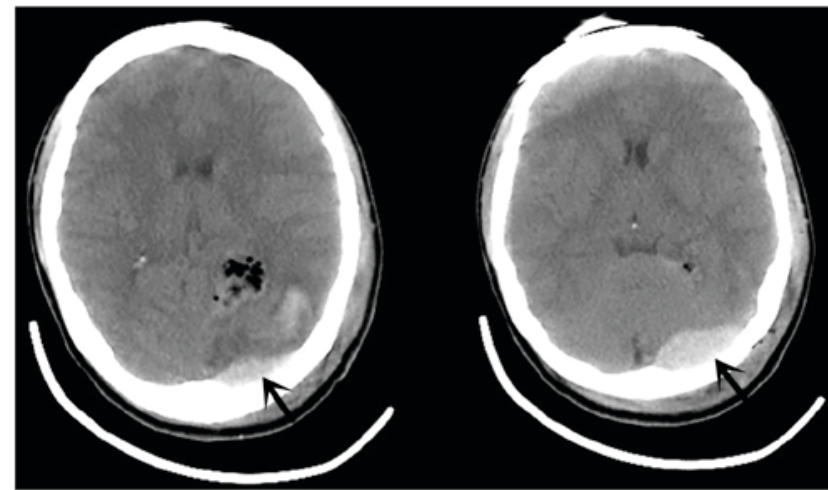

Figure 4. Computed tomography of the patient 1 day after initial surgery indicating complete resection of the tumor. An infratentorial epidural hematoma was detected near the surgery area (indicated by arrow).

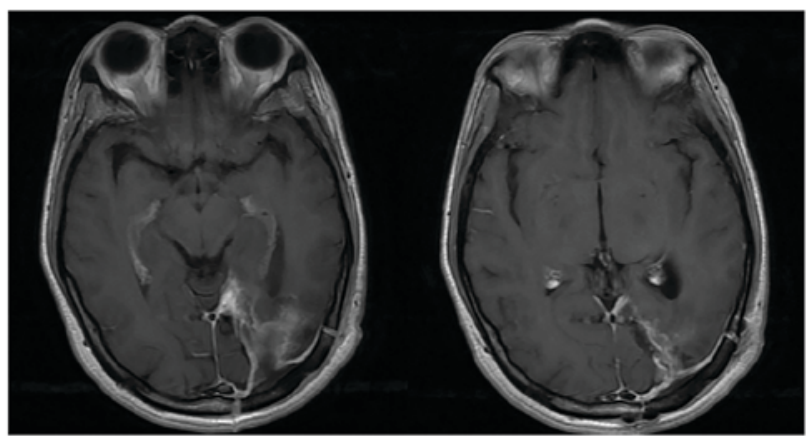

Figure 5. Post-gadolinium axial T1-weighted image from the scan performed on the patient $\sim 2$ months following initial surgery. No recurrence of the tumor was detected and the surgical pathway through the cortex is clearly presented.

and capillary hemangioma' on Pubmed (https://www.ncbi .nlm.nih.gov/pubmed/) and 19 articles were identified reporting 29 cases of $\mathrm{ICH}$ that were histologically confirmed (Table I).

The 29 cases included 14 male and 15 female patients with $\mathrm{ICH}$ ranging in age from 2 weeks to 69 years. The avaliable data were limited, however there were no significant differences in cases based on sex. Data regarding age demonstrated that the majority of patients were infants (7) and young adults $<30$ years old. Furthermore, only one case of ICH was reported prior to 2000 (9). One possible reason for this is that similar cases may have been described using other terms of pathological diagnosis (4). Due to greater in-depth knowledge of ICH gleaned from pathology reports, an increasing number of cases have been identified more recently. However, accurately diagnosing all cases of ICH is nearly impossible due to the use of non-standardized nomenclature to describe intracranial vascular lesions. One case with mixed capillary and cavernous hemangioma histology was included in the current study (10), however other similar cases may not have been correctly diagnosed and therefore were not correctly reported. Four cases of intraosseous $\mathrm{CH}$ were not included (11-14).

Despite the fact that $\mathrm{ICH}$ exhibits consistant pathological features, the clinical characteristics of the disease exhibit a high degree of heterogeneity. ICH can grow in the cerebral lobe $(4,9)$, cavernous sinus $(15)$, lumen of the superior sagittal 


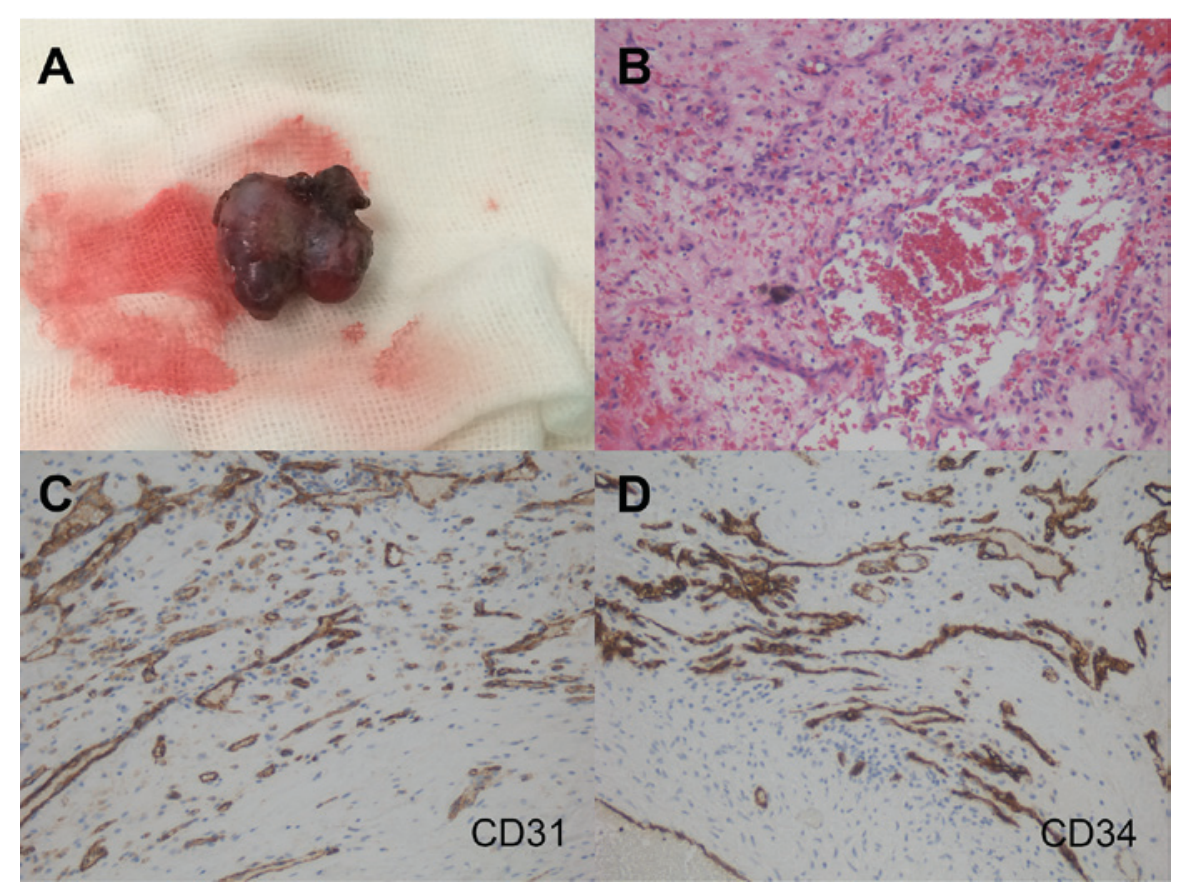

Figure 6. Histopathological observations of the tumor itself. (A) The tumor was completely resected as a dark-red, firm, solid, $2.2 \times 1.6 \times 1.7 \mathrm{~cm}$ mass with a broad-based dural attachment. (B) Photomicrographs of the surgical specimen identified the area encompassed by the tumor with moderate-to-dense cellularity containing numerous vascular channels and spaces. Hematoxylin and eosin staining, magnification x100. (C and D) Vascular structures and the endothelial cell lining of this tumor exhibited high expression of the vascular markers CD31 and CD34. CD31 and CD34 immunohistochemical staining, magnification x100.

sinus (16), cerebellum (4), anterior choroidal artery (17), fourth venticle (8) and sellar region (18). Following analysis of the 29 cases, no predilection for a specific site of ICH growth was apparent and in a number of cases, only a single lesion was detected but there were multiple tumors (4 of 29 reported cases) $(4,19,20)$. The clinical symptoms of ICH are very diverse and differ according to tumor location and size. All reported cases in the literature were symptomatic and more common symptoms include headaches, cranial nerve palsy and/or seizures. In 4 of the previously reported cases, ICH was associated with pregnancy $(2,21,22)$. By contrast, the patient in the current study was nearly asymptomatic and visited the hospital due to a slight but recurring morning headache that increased in intensity. However, these headaches spontaneously resolved without any treatment; therefore, the patient experienced no marked symptoms associated with $\mathrm{ICH}$, aside from anxiety, prior to initial surgery. An intracranial lesion was detected by neuroimaging examination, however it could not be confirmed that the headaches were associated with the lesion.

The patient had last been pregannt 5 years ago, which resulted in mild hypertension and the patient's blood pressure was controlled by oral medication. The tumor may have been associated with this pregnancy and may have therefore been present for several years. The tumor that was resected during surgery was solid and small and may have been in an involution phase by the time it was detected. All these factors further support the hypothesis that the incidence of ICH may be underreported, particularly if the tumor regresses in size over time and remains asymptomatic.

Mutiple differential diagnoses were noted in previous studies but there was limited information avaliable to guide preoperative diagnosis. Due to differences in tumor location and size, differential diagnoses made in one article could not be directly applied to other cases. Capillary hemangioma was diagnosed by pathological observations in all cases. However, ICH was not considered in preoperative differential diagnoses. In the present case, a tentorium-based lesion mimicking a meningioma was identified, which was preoperatively diagnosed as an atypical meningioma. Astrocytoma was also considered, with no expectation of the final result. Therefore, preoperatively diagnosing ICHs is difficult.

Treatment for ICH is empirical (22). Different modalities, including surgery, stereotactic radiotherapy, or treatment with corticosteroids or interferon have been used; however, the rationale and indications for different treatments have not been clearly stated in the literature. The vast majority of patients (28 of 29) underwent surgery, among which there were 24 resections and 4 biopsies. A capacity for rapid growth of the lesion was reported and the turmor recurred after a short time in three cases $(2,7,18)$. In symptomatic cases, performing surgery is a less controversial option, however performing surgery in asymptomatic cases should be considered more carefully. Theoretically, conservative observations should be conducted on a regular basis. Taking the final histopathological results into account, it may have been more appropriate for the current patient to undergo conservative observation; however, a tumor that is suspected to be serious requires a more radical approach, such as surgery. In the current case report, the patient refused conservative observation and requested radical surgery as soon as possible. The outcomes of the 29 patients included in the literature review were generally satisfactory, with only one case of mortality and 3 definite recurrences and no more severe neurological defects. More details are shown in Table I.

In conclusion, the present study reported a nearly asymptomatic case of ICH. ICHs are rare entities defined by highly 


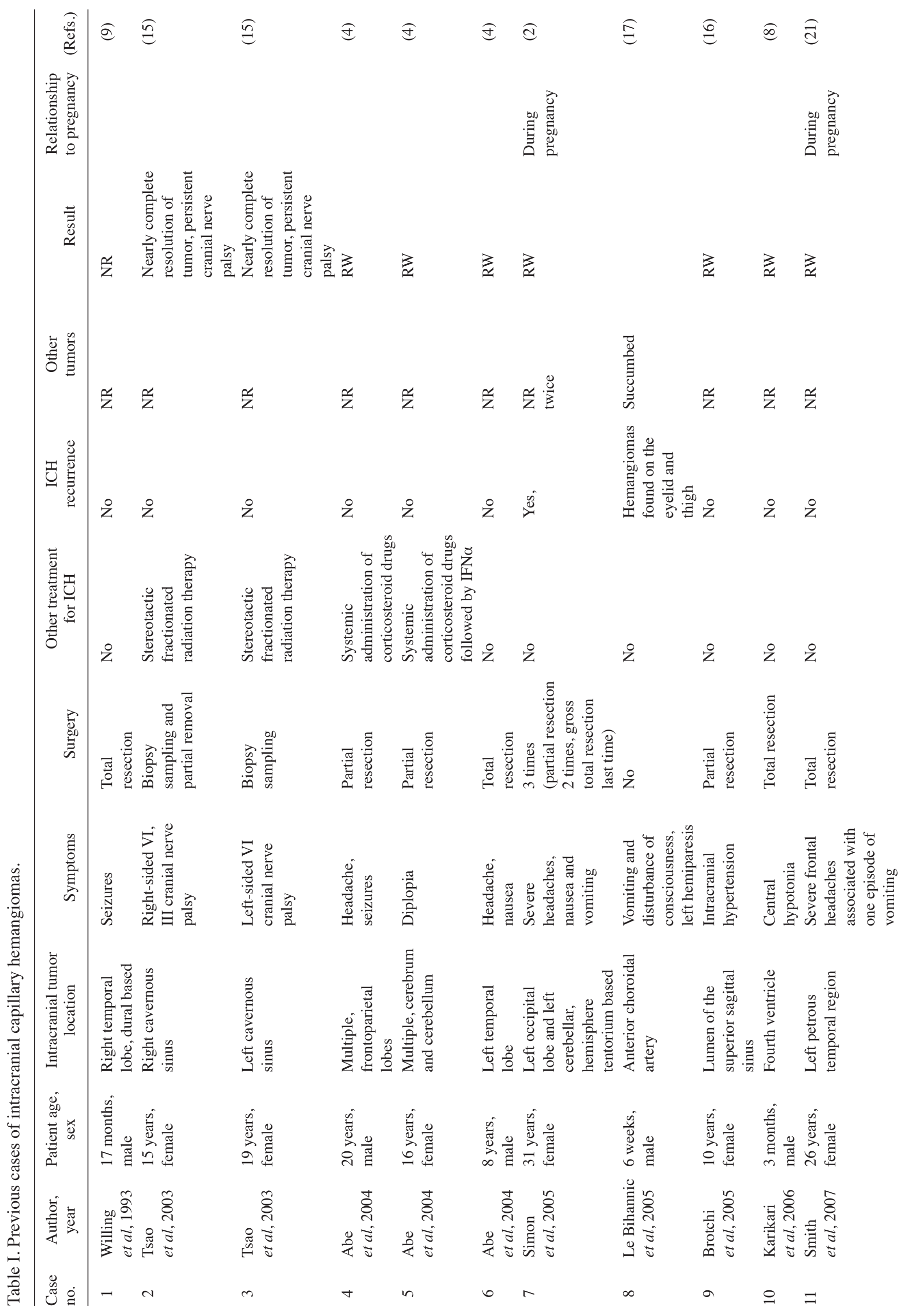




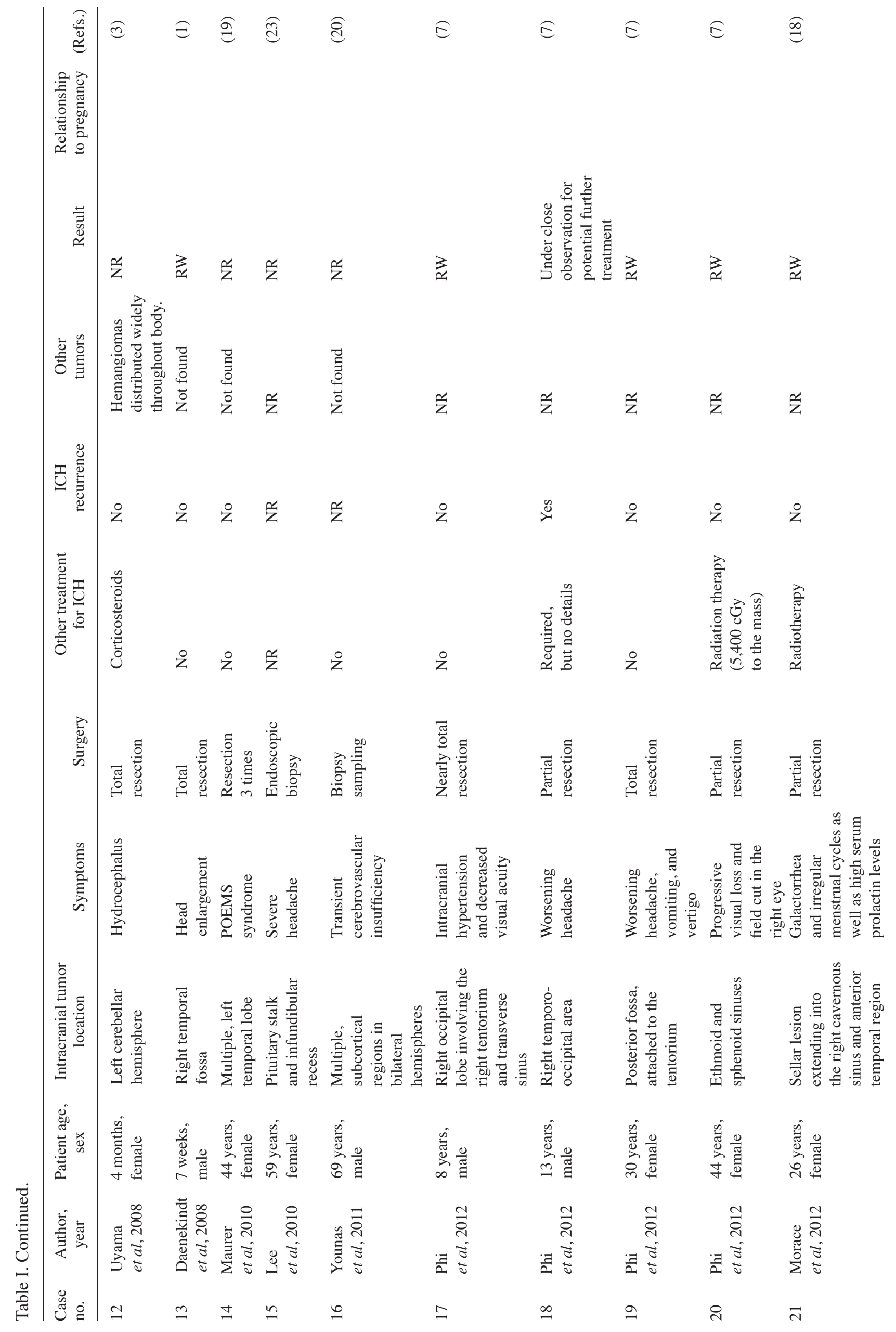




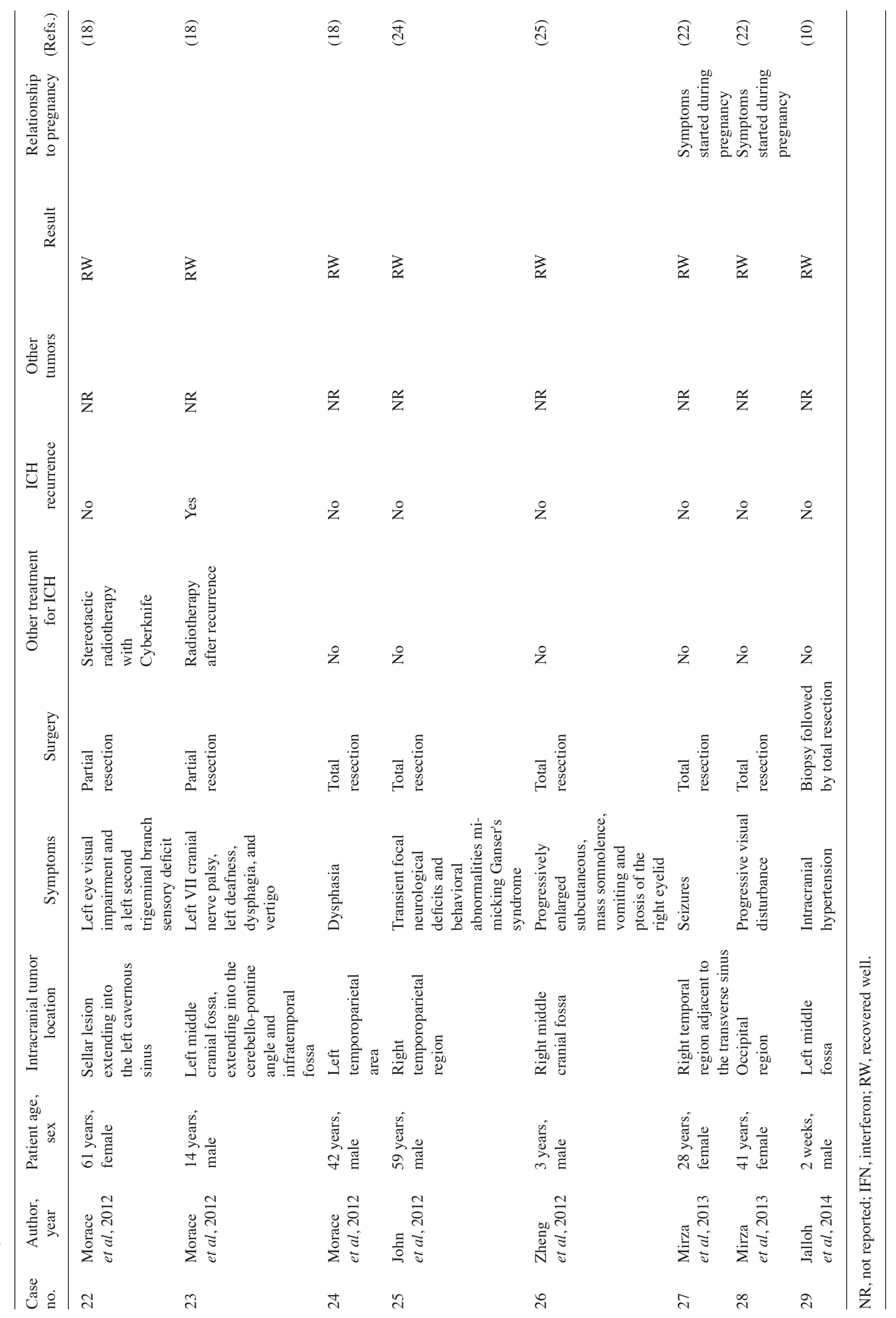


consistent histopathological results but exhibit variable clinical features. The clinical symptoms of ICH are diverse and largely depend on tumor location and size and preoperative diagnosis are difficult to make. Treatment for ICH remains empirical but surgery is the most common method and patients undergoing surgery generally have good prognoses. Although rapid growth and recurrence or massive bleeding during surgery have been reported, the majority of patients achieve long-term survival with no further symptomatic events and no tumor recurrence.

\section{References}

1. Daenekindt T, Weyns F, Kho KH, Peuskens D, Engelborghs K and Wuyts J: Giant intracranial capillary hemangioma associated with enlarged head circumference in a newborn. J Neurosurg Pediatr 1: 488-492, 2008.

2. Simon SL, Moonis G, Judkins AR, Scobie J, Burnett MG, Riina HA and Judy KD: Intracranial capillary hemangioma: Case report and review of the literature. Surg Neurol 64: 154-159, 2005.

3. Uyama A, Kawamura A, Akiyama H, Nakamizo S, Yamamoto K, Nagashima T, Uetani T, Takeda $\mathrm{H}$ and Yoshida M: A case of cerebellar capillary hemangioma with multiple cysts. Pediatr Neurosurg 44: 344-349, 2008.

4. Abe M, Tabuchi K, Tanaka S, Hodozuka A, Kunishio K, Kubo N and Nishimura Y: Capillary hemangioma of the central nervous system. J Neurosurg 101: 73-81, 2004.

5. Pignotti F, Coli A, Fernandez E and Montano N: Capillary hemangioma of the cauda equina. Surg Neurol Int 6: 133, 2015.

6. Roncaroli F, Scheithauer BW and Krauss WE: Hemangioma of spinal nerve root. J Neurosurg 91 (2 Suppl): S175-S180, 1999.

7. Phi JH, Kim SK, Cho A, Kim DG, Paek SH,Park SH and Wang KC: Intracranial capillary hemangioma: Extra-axial tumorous lesions closely mimicking meningioma. J Neurooncol 109: 177-185, 2012.

8. Karikari IO, Selznick LA, Cummings TJ and George TM: Capillary hemangioma of the fourth ventricle in an infant. Case report and review of the literature. J Neurosurg 104 (3 Suppl): S188-S191, 2006.

9. Willing SJ, Faye-Petersen O, Aronin P and Faith S: Radiologic-pathologic correlation. Capillary hemangioma of the meninges. AJNR Am J Neuroradiol 14: 529-536, 1993.

10. Jalloh I, Dean AF, O'Donovan DG, Cross J, Garnett MR and Santarius T: Giant intracranial hemangioma in a neonate. Acta Neurochir (Wien) 156: 1151-1154, 2014.
11. Shah ZK, Peh WC, Shek TW, Wong JW and Chien EP: Hemangioendothelioma with an epithelioid phenotype arising in hemangioma of the fibula. Skeletal Radiol 34: 750-754, 2005.

12. Frei-Jones M, McKinstry RC, Perry A, Leonard JR, Park TS and Rubin JB: Use of thalidomide to diminish growth velocity in a life-threatening congenital intracranial hemangioma. J Neurosurg Pediatr 2: 125-129, 2008.

13. Suss RA, Kumar AJ, Dorfman HD, Miller NR and Rosenbaum AE: Capillary hemangioma of the sphenoid bone. Skeletal Radiol 11: 102-107, 1984.

14. Yang G, Li C, Chen X, Liu Y, Han D, Gao X, Kawamoto K and Zhao S: Large capillary hemangioma of the temporal bone with a dural tail sign: A case report. Oncol Lett 8: 183-186, 2014.

15. Tsao MN, Schwartz ML, Bernstein M, Halliday WC, Lightstone AW, Hamilton MG, Jaywant $\mathrm{S}$ and Laperriere N: Capillary hemangioma of the cavernous sinus. Report of two cases. J Neurosurg 98: 169-174, 2003.

16. Brotchi J, Baleriaux D, Kalangu KK, Morelli D, Rodesch G, Rorive S and Pirotte B: Capillary hemangioma in the superior sagittal sinus as a rare cause of intracranial hypertension in a child: Case report. Neurosurgery 57: E815, 2005.

17. Le Bihannic A, Michot C, Heckly A, Loget P, Beucher A, Brassier G and Hamlat A: Capillary haemangioma arising from the anterior choroidal artery. Childs Nerv Syst 21: 265-271, 2005.

18. Morace R, Marongiu A, Vangelista T, Galasso V, Colonnese C, Giangaspero F, Innocenzi G, Esposito V and Cantore G: Intracranial capillary hemangioma: A description of four cases. World Neurosurg 78: 191.E15-E21, 2012.

19. Maurer GD, Schittenhelm J, Ernemann U, Kempf VA, Ritz R, Weller $\mathrm{M}$ and Schmidt F: Intracranial hemangiomas in a patient with POEMS syndrome. J Neurol 257: 484-487, 2010.

20. Younas F, Durrani Q, Shahzad MA, Mushtaq A and Imlay S: Multiple intracranial capillary hemangiomas and transient cerebrovascular insufficiency. Neurol Sci 32: 963-966, 2011.

21. Smith IF and Skelton V: An unusual intracranial tumour presenting in pregnancy. Int J Obstet Anesth 16: 82-85, 2007.

22. Mirza B, Shi WY, Phadke R, Holton JL, Turner C, Plant GT, Brew S, Kitchen N and Zrinzo L: Strawberries on the brain-intracranial capillary hemangioma: Two case reports and systematic literature review in children and adults. World Neurosurg 80: 900.e13-e21, 2013.

23. Lee YH, Park YS, Kim DS, Park YG and Shim KW: 59-year old female with suprasellar mass. Brain Pathol 20: 257-260, 2010.

24. John SG, Pillai U and Lacasse A: Intracranial capillary hemangioma mimicking a dissociative disorder. Clin Pract 2: e35, 2012.

25. Zheng SP, Ju Y and You C: Giant intracranial capillary hemangioma in a 3-year-old child: Case report and literature review. Clin Neurol Neurosurg 114: 1270-1273, 2012. 